\title{
Rational Polygons: Odd Compression Ratio and Odd Plane Coverings*
}

\author{
Rom Pinchasi ${ }^{\dagger} \quad$ Yuri Rabinovich ${ }^{\ddagger}$
}

November 15, 2018

\begin{abstract}
Let $P$ be a polygon with rational vertices in the plane. We show that for any finite oddsized collection of translates of $P$, the area of the set of points lying in an odd number of these translates is bounded away from 0 by a constant depending on $P$ alone.

The key ingredient of the proof is a construction of an odd cover of the plane by translates of $P$. That is, we establish a family $\mathcal{F}$ of translates of $P$ covering (almost) every point in the plane a uniformly bounded odd number of times.
\end{abstract}

\section{Introduction}

The starting point of this research is the following isoperimetric-type problem about translates of compact sets in $\mathbb{R}^{d}$ :

Let $X \subset \mathbb{R}^{d}$ be a compact set, and let $Z \subset \mathbb{R}^{d}$ be a finite set of odd cardinality. Consider the finite odd-sized collection $\mathcal{F}=\{X+z\}_{z \in Z}$ of translates of $X$. Let $U \subset \mathbb{R}$ be the set of all points that belong to an odd number of the members of $\mathcal{F}$. How small can be the Lebesgue measure of $U$ in terms of the Euclidean measure of $X$ ?

Denoting the infimum of this value by $\operatorname{Vol}_{\text {odd }}(X)$, called the odd volume of $X$, we define the odd compression ratio of $X$ as $\alpha^{\circ}(X)=\operatorname{Vol}_{\text {odd }}(X) / \operatorname{Vol}(X)$, where $\operatorname{Vol}(X)$ is the Euclidean volume of $X$. Observe that $\alpha^{\circ}(X) \leq 1$, as $\mathcal{F}$ may consist of a single element $X$. Clearly, $\alpha^{\circ}(X)$ is an affine invariant.

It was observed by the second author about a decade ago that $\alpha^{\circ}$ of a unit $d$-cube $Q^{d}$ is 1 . Indeed, informally, consider $\mathbb{R}^{d}$ under the action (i.e., translation) of $\mathbb{Z}^{d}$. The unit cube (with parts of its boundary removed) is a fundamental domain of $\mathbb{R}^{d} / \mathbb{Z}^{d}$. The quotient map $\phi: \mathbb{R}^{d} \rightarrow Q^{d}$ maps any translate of $Q^{d}$ onto $Q^{d}$ in a one-to-one manner. Moreover, the quotient map satisfies

$$
\phi\left(\bigoplus_{\mathcal{T} \in \mathcal{F}} T\right)=\bigoplus_{\mathcal{T} \in \mathcal{F}} \phi(T),
$$

${ }^{*}$ The final version of the contribution is due to be published in the collection of papers A Journey through Discrete Mathematics: A Tribute to Jiri Matousek edited by Martin Loebl, Jaroslav Nesetril and Robin Thomas, due to be published by Springer.

${ }^{\dagger}$ Math. Department, Technion-Israel Institute of Technology, Haifa 32000, Israel. room@math.technion.ac.il. Supported by ISF grant (grant No. 409 /16)

${ }^{\ddagger}$ Department of Computer Science, Haifa University, Haifa, Israel. yuri@cs.haifa.ac.il. 
where $\bigoplus$ denotes the set-theoretic union modulo 2 , i.e., the set of all points covered by an odd number of the members of $\mathcal{F}$. Since the quotient map is locally volume preserving, it is globally volume-nondecreasing, and so one concludes that the volume of $\bigoplus_{\mathcal{T} \in \mathcal{F}} T$ is at least that of $\phi\left(\bigoplus_{\mathcal{T} \in \mathcal{F}} T\right)=\bigoplus_{\mathcal{T} \in \mathcal{F}} \phi(T)=\bigoplus_{\mathcal{T} \in \mathcal{F}} Q^{d}=Q^{d}$

A similar argument shows that $\alpha^{\circ}$ of a centrally symmetric planar hexagon is 1 as well. But what about other sets, i.e., a triangle? The second author vividly remembers discussing this question with Jirka Matoušek in a pleasant cafe at Malà Strana, laughing that they are too old for Olympiad-type problems...1

The value of $\alpha^{0}$ of the triangle (recall that any two triangles are affinely equivalent) was determined by the first author in [1]; it is $\frac{1}{2}$.

Next significant progress on the problem was obtained in [2]. It was shown there that for a union of two disjoint intervals of length 1 on a line with a certain irrational distance between them, the odd compression ratio is 0 . The proof uses some algebra of polynomials, and Diophantine approximation. The construction easily extends to higher dimensions. In addition, 2] introduced a technique for obtaining lower bounds on $\alpha^{\circ}(X)$, and used it to show that for $X^{\prime}$ 's that are unions of finitely many cells of the 2-dimensional grid, $\alpha^{\circ}(X)>0$.

In the present paper we further develop the technique of [2], and use it to prove that for any planar rational polygon $P$, the odd compression ratio $\alpha^{\circ}(P)$ is bounded away from 0 by some positive constant explicitly defined in terms of $P$. In fact, the statement applies to any compact planar figure with piecewise linear boundary, and (finitely many) rational vertices. In view of the above mentioned result from [2], the assumption of rationality cannot in general be dropped.

Perhaps more importantly, the value of $\alpha^{\circ}(X)$ is related here to the value of some other natural geometric invariant of $X$. The other invariant is $\theta^{\circ}(X)$, the smallest possible average density in an odd cover of $\mathbb{R}^{2}$ by a family $\mathcal{F}$ of translates of $X$. By odd cover we mean that every point $p \in \mathbb{R}^{2}$, with a possible exception of a measure 0 set, is covered by the members of $\mathcal{F}$ an odd and uniformly bounded number of times.

While [2] does not directly consider odd covers of $\mathbb{R}^{2}$, it still implies that $\alpha^{\circ}(X) \geq \theta^{\circ}(X)^{-1}$. We include here two complete proofs of this useful inequality.

The existence of odd covers of $\mathbb{R}^{2}$ by translates of a rational polygon is by no means obvious. Most of the present paper is dedicated to constructing such covers. We are aware of no related results in the literature.

While many of the results and constructions presented here can be easily extended to higher dimensions, some essential parts resist simple generalization, and more work is required in order to understand the situation there.

To conclude the Introduction, we hope that the present paper will somewhat elucidate the meaning of the odd compression ratio $\alpha^{\circ}(X)$, and that the odd covers introduced here will prove worthy of further study.

\section{Preliminaries}

\subsection{Two Basic Operators}

In what follows, we shall extensively use the following two operators on subsets of $\mathbb{R}^{2}$ : $\oplus$ and $\stackrel{\circ}{+}$ Let us briefly discuss them here.

The first operator $\oplus$ is the set-theoretic union modulo 2 . Given a family $\mathcal{F}$ of subsets of $\mathbb{R}^{2}$ so

\footnotetext{
${ }^{1} \mathrm{~A}$ discrete version of the problem about the translates of a square in $\mathbb{R}^{2}$ had indeed found its way into a mathematical olympiad [4.
} 
that any $p \in \mathbb{R}^{2}$ is covered at most finitely many times by $\mathcal{F}, \bigoplus_{X \in \mathcal{F}} X$ is the set of all points of $\mathbb{R}^{2}$ covered by an odd number of members in $\mathcal{F}$. Observe that $\oplus$ is commutative and associative.

The second operator, $\stackrel{+}{+}$, is less standard. It is the Minkowski sum modulo 2 :

$$
X+Z=\bigoplus_{x \in X, z \in Z} x+z=\bigoplus_{z \in Z}(X+z),
$$

where $X+z$ denotes the translate of $X$ by $z$. I.e., $a \in X+Z$ if and only if the number of representations of $a$ of the form $a=x+z$ is an odd natural number. Unlike the Minkowski sum, $X+Z$ is well defined only when every $a \in \mathbb{R}^{2}$ has at most finitely many representations of the form $x+z$ as above. This requirement is met, e.g., when $Z$ is finite, or when $Z$ is a discrete set of points at distance $\geq \epsilon>0$ from each other, and $X$ is bounded. Since the Minkowski sum extends to any

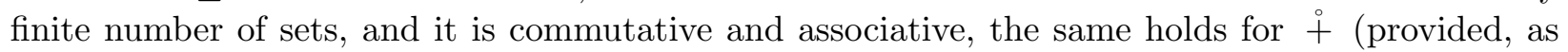
before, that every $a$ has finitely many representations).

Moreover, the following distributive law holds. Let $\mathcal{G}$ be a family of sets in $\mathbb{R}^{2}$, and let $S \subset \mathbb{R}^{2}$. Assume that the family of sets $\{Y+s\}_{Y \in \mathcal{G}, s \in S}$ covers any point of $\mathbb{R}^{2}$ at most finitely many times. Then:

$$
\left(\bigoplus_{Y \in \mathcal{G}} Y\right) \stackrel{\circ}{+}=\bigoplus_{Y \in \mathcal{G}}(Y \stackrel{\circ}{+} S) \text {. }
$$

Indeed, the equality is trivial when $S$ consists of a single element. Thus, by definition of $\stackrel{\circ}{+}$,

$$
\left(\bigoplus_{Y \in \mathcal{G}} Y\right)+\stackrel{+}{+}=\bigoplus_{s \in S}\left(\left(\bigoplus_{Y \in \mathcal{G}} Y\right)+s\right)=\bigoplus_{s \in S} \bigoplus_{Y \in \mathcal{G}}(Y+s) \stackrel{*}{=} \bigoplus_{Y \in \mathcal{G}} \bigoplus_{s \in S}(Y+s)=\bigoplus_{Y \in \mathcal{G}}(Y \stackrel{+}{+} S) .
$$

It remains to validate the change of order of summation in the starred equality. For $a \in \mathbb{R}^{2}$ consider the set $\{(Y, s) \mid a \in Y+s\} \subseteq \mathcal{G} \times S$. By our assumptions, this set is always finite. Therefore, for any $a, \mathbf{1}_{a}\left(\bigoplus_{s \in S} \bigoplus_{Y \in \mathcal{G}}(Y+s)\right)=\bigoplus_{s \in S} \bigoplus_{Y \in \mathcal{G}} \mathbf{1}_{a}(Y+s)$ has only finitely many nonzero terms. Hence, the order of summation in the double sum $\bigoplus_{s \in S} \bigoplus_{Y \in \mathcal{G}}(Y+s)$ is interchangeable.

Finally, notice that similarly to Minkowski sum, $X \stackrel{+}{+}=\emptyset$, while $X \oplus \emptyset=X$.

\subsection{Covers and Their Densities}

It is important to stress that throughout this paper whenever we speak on covers or odd covers of the plane it always means covering up to a set of measure 0 , even if it is not explicitly said so. This convention helps to avoid discussing unnecessary technicalities related to the boundaries of the sets in the cover.

For every compact measurable set $X \subset \mathbb{R}^{2}$, we denote by $A(X)$ the Euclidean area of $X$. Let $Z \subseteq \mathbb{R}^{2}$ be a discrete set. The family $\mathcal{F}=\{X+z\}_{z \in Z}$ has a uniformly bounded degree if there exists a constant $d_{\mathcal{F}}$ such that every $a \in \mathbb{R}^{2}$ belongs to at most $d_{\mathcal{F}}$ members of $\mathcal{F}$. Further, such $\mathcal{F}$ is called a cover of $\mathbb{R}^{2}$ if $X+Z=\mathbb{R}^{2}$. I.e., the cover degree of any $a \in \mathbb{R}^{2}$ by the members of a cover $\mathcal{F}$ is uniformly bounded, and, up to a set of measure 0 , it is strictly positive.

The (lower) density of $\mathcal{F}$ with a uniformly bounded degree, $\rho(\mathcal{F})$, is defined by

$$
\rho(\mathcal{F})=\liminf _{n \rightarrow \infty} \frac{\sum_{z \in Z} A\left(Q_{n} \cap(X+z)\right)}{n^{2}},
$$

where $Q_{n}$ is the $n \times n$ square centered at the origin. Clearly, $\rho(\mathcal{F}) \geq 1$ when $\mathcal{F}$ is a cover or $\mathbb{R}^{2}$.

Since $\sum_{z \in Z} A\left(Q_{n} \cap X+z\right) / n^{2}$ is precisely the average of the cover degrees $d_{\mathcal{F}}(a)$ where $a$ ranges over $Q_{n}$, the density $\rho(\mathcal{F})$ can be viewed as a kind of an average degree of the cover of $\mathbb{R}^{2}$ by $\mathcal{F}$. 
Claim 2.1. Fixing $Z$ and varying the (measurable) $X$, the density of the family $\mathcal{F}=\{X+z\}_{z \in Z}$ is proportional to $A(X)$. I.e., $\rho(\mathcal{F})=c_{Z} \cdot A(X)$, where $c_{Z}$ is a constant depending solely on $Z$. (In particular, when $Z$ is a lattice, $c_{Z}$ is the reciprocal of the area of the fundamental domain of Z.)

Proof. (Sketch) Assume w.l.o.g., that $X$ contains the origin, and let $\delta=\operatorname{Diam}(X)$. Consider $\Delta_{n}=\left|\sum_{z \in Z} A\left(Q_{n} \cap X+z\right)-\right| Z \cap Q_{n}|\cdot A(X)|$. How big can it be? On the one hand,

$$
\left|Z \cap Q_{n-2 \delta}\right| \cdot A(X) \leq \sum_{z \in Z} A\left(Q_{n} \cap X+z\right) \leq\left|Z \cap Q_{n+2 \delta}\right| \cdot A(X)
$$

and therefore

$$
\Delta_{n} \leq\left|Z \cap Q_{n+2 \delta}\right| \cdot A(X)-\left|Z \cap Q_{n-2 \delta}\right| \cdot A(X)=\left|Z \cap\left(Q_{n+2 \delta} \backslash Q_{n-2 \delta}\right)\right| \cdot A(X) .
$$

On the other hand, since $Z \cap\left(Q_{n+2 \delta} \backslash Q_{n-2 \delta}\right)+X$ is contained in $Q_{n+4 \delta} \backslash Q_{n-4 \delta}$, covering no point there more than $d_{\mathcal{F}}$ times, it follows that $\left|Z \cap\left(Q_{n+2 \delta} \backslash Q_{n-2 \delta}\right)\right| \cdot A(X)$ is at most $O(n) \cdot \delta \cdot d_{\mathcal{F}}$. Hence, $\Delta_{n}=O(n) \cdot \delta \cdot d_{\mathcal{F}}$, and so $\Delta_{n} / n^{2} \rightarrow 0$. The conclusion follows:

$$
\begin{gathered}
\rho(\mathcal{F})=\liminf _{n \rightarrow \infty} \frac{\sum_{z \in Z} A\left(Q_{n} \cap X+z\right)}{n^{2}}=\liminf _{n \rightarrow \infty} \frac{\left|Z \cap Q_{n}\right| \cdot A(X) \pm \Delta_{n}}{n^{2}}= \\
\liminf _{n \rightarrow \infty} \frac{\left|Z \cap Q_{n}\right|}{n^{2}} \cdot A(X)=c_{Z} \cdot A(X) .
\end{gathered}
$$

The fact that for a lattice $Z, \lim _{n \rightarrow \infty}\left|Z \cap Q_{n}\right| / n^{2}$ is the inverse of the the area of the fundamental domain of $Z$, is well known (see, e.g., [3]).

The covering density of $X, \theta(X) \geq 1$, is defined as the infimum of $\rho(\mathcal{F})$ over all covers of the form $\mathcal{F}=\{X+z\}_{z \in Z}$. It is well known (see, e.g., [3]) that $\theta(X)$ is an affine invariant.

\subsection{Odd Covers}

Let $X \subset \mathbb{R}^{2}$ be a compact set of a positive area $A(X)>0$. The family $\mathcal{F}=\{X+z\}_{z \in Z}$ for $Z \subseteq \mathbb{R}^{2}$ is called an odd cover of $\mathbb{R}^{2}$ if $X+Z$ is well defined, and equals to $\mathbb{R}^{2}$ up to a set of measure 0 . Notice that if $\mathcal{F}$ is an odd cover of $\mathbb{R}^{2}$, then in particular it is a cover of $\mathbb{R}^{2}$. As before, we shall further require that the maximal degree of the cover of $\mathbb{R}^{2}$ by $\mathcal{F}$ is uniformly bounded.

The odd covering density of a compact $X, \theta^{\circ}(X) \geq 1$ is defined as the infimum of $\rho(\mathcal{F})$ over all odd covers $\mathcal{F}$ as above. If no such $\mathcal{F}$ exists, set $\theta^{\circ}(X)=\infty$. Notice that $\theta^{\circ}(X) \geq \theta(X)$. Similarly to the usual covering density $\theta(X)$, the odd covering density $\theta^{\circ}(X)$ is an affine invariant. This intuitively plausible statement can be proved formally along the same lines as the standard proof of the corresponding statement for the usual covers (see, e.g., [3]). ${ }^{2}$

\footnotetext{
${ }^{2}$ The requirement that $\mathcal{F}$ has a uniformly bounded degree does not appear in the standard definition of $\theta(X)$, despite the fact that it is used in the proof of the affine invariance of $\theta(X)$ and elsewhere. The reason is that for any $\epsilon>0$, a cover $\mathcal{F}$ can be easily modified into a periodic cover $\mathcal{F}^{\prime}$ with $\rho\left(\mathcal{F}^{\prime}\right) \leq \rho(\mathcal{F})+\epsilon$, i.e., the corresponding $Z^{\prime}$ is of the form $\Lambda+K$, where $\Lambda$ is a lattice, and $K$ is finite (see, e.g., [3]). Thus, w.l.o.g., one may restrict the discussion of $\theta(X)$ to periodic covers, and those are always uniformly bounded for a compact $X$. In contrast, the odd covers apparently do not allow such a modification, and so the assumption about the uniformly bounded degree seems to be essential for them. This said, all odd covers occurring in this paper are periodic.
} 


\subsection{Odd Compression Ratio: the Definition}

Let $X \subset \mathbb{R}^{2}$ be a compact set of area $0<A(X)<\infty$. Define $A_{\text {odd }}(X)$, the odd area of $X$, to be the maximum number such that for any finite and odd-sized collection $\mathcal{F}$ of translates of $X$, the set of all points in $\mathbb{R}^{2}$ belonging to an odd number of members of $\mathcal{F}$ has area $\geq A_{\text {odd }}(X)$. I.e., $A_{\text {odd }}(X)$ is the infimum of $A(X+K)$ over all finite odd-sized sets $K \subset \mathbb{R}^{2}$ (see [1, 2]).

Define $\alpha^{\circ}(X)$, the odd compression ratio of $X$, as $A_{\text {odd }}(X) / A(X)$. Clearly, $0 \leq \alpha^{\circ}(X) \leq 1$, and it is an affine invariant.

\section{The Odd Cover Lemma}

The following lemma, a variant, and in fact a special case, of Lemma 1 from [2], is a useful tool for obtaining lower bounds on the odd compression ratio of $X$. For completeness, we provide two different proofs for it. The first is shorter and simpler due to the preparation done in Section 2.2. It is a streamlined variant of the proof used in [2]. The second proof follows a somewhat different logic, and can be viewed as a generalization of the factor-space argument mentioned in the Introduction.

Lemma 3.1. For any compact set $X$ of a positive measure in $\mathbb{R}^{2}$, the odd compression ratio of $X$ is at least the reciprocal of its odd covering density. That is,

$$
\alpha^{\circ}(X) \geq \theta^{\circ}(X)^{-1}
$$

Proof. (A) Let $\mathcal{F}=\{X+z\}_{z \in Z}$ be an odd cover of $\mathbb{R}^{2}$ of density $\rho(\mathcal{F})$, and maximal cover degree $d_{\mathcal{F}}<\infty$. (If no such $\mathcal{F}$ exists, the lemma is trivially true.) Let $K \subset \mathbb{R}^{2}$ be any finite set of odd cardinality. Set $Y=X+K$.

Consider the set $\left(X \stackrel{+}{+} \stackrel{\circ}{+} K\right.$. On the one hand, it is equal to $\mathbb{R}^{2}$, up to a set of measure 0 . This is because $(X+Z)=\mathbb{R}^{2}$, again up to a set of measure 0 , and the cardinality of $K$ is odd.

On the other hand, using the commutativity of $\stackrel{\circ}{+}$, one concludes that $(X+Z)+K=$ $(X \stackrel{+}{+}) \stackrel{+}{+}=Y \stackrel{+}{ }$. In other words, the family $\mathcal{G}=\{Y+z\}_{z \in Z}$ is an odd cover of $\mathbb{R}^{2}$ of a maximal covering degree at most $d_{\mathcal{F}} \cdot|K|$.

By Claim 2.1, there is a constant $c_{Z}$ depending only on $Z$, such that for every measurable set $W \subset \mathbb{R}^{2}$ such that $\{W+z\}_{z \in Z}$ is a cover of $\mathbb{R}^{2}$, it holds that $\rho\left(\{W+z\}_{z \in Z}\right)=c_{Z} \cdot A(W)$. Therefore,

$$
1 \leq \rho(\mathcal{G})=c_{Z} \cdot A(Y)=\rho(\mathcal{F}) \cdot \frac{A(Y)}{A(X)} \quad \Longrightarrow \quad \rho(\mathcal{F})^{-1} \leq \frac{A(Y)}{A(X)}
$$

Taking the infimum over all odd-sized $K$ 's to minimize $A(Y) / A(X)$, and the infimum over all legal $Z$ 's to minimize $\rho(\mathcal{F})$, one concludes that $\theta^{\circ}(X)^{-1} \leq \alpha^{\circ}(X)$.

Proof. (B) Let $\mathcal{F}=\{X+z\}_{z \in Z}$ be an odd cover of $\mathbb{R}^{2}$ as before, and let $S \subset \mathbb{R}^{2}$ be compact. Consider the following mapping $\phi$ of the compact sets $S$ to the compact subsets of $X$ :

$$
\phi(S)=\bigoplus_{z \in Z}(S-z) \cap X=(S+(-Z)) \cap X .
$$

\section{Claim 3.1.}

1. $\phi(-X+a)=X$;

2. $\phi\left(\bigoplus_{i=1}^{k} S_{i}\right)=\bigoplus_{i=1}^{k} \phi\left(S_{i}\right)$; 
3. $A(\phi(S)) \leq A(S) \cdot \tilde{d}_{\mathcal{F}}(S)$, where $\tilde{d}_{\mathcal{F}}(S)$ is the average degree of a cover of $S$ by $\mathcal{F}$, i.e., the average of the cover degrees $d_{\mathcal{F}}(a)$, where a ranges over $S$.

Proof. Indeed, for (1), keeping in mind that $X \stackrel{+}{+}=\mathbb{R}^{2}$, and that $a-\mathbb{R}^{2}=\mathbb{R}^{2}$, one gets

$\phi(-X+a)=\bigoplus_{z \in Z}(-X+a-z) \cap X=X \cap \bigoplus_{z \in Z} a+(-X-z)=X \cap a-(X+Z)=X \cap \mathbb{R}^{2}=X$.

For $(2)$,

$\phi\left(\bigoplus_{i=1}^{k} S_{i}\right)=\bigoplus_{z \in Z}\left(\left(\bigoplus_{i=1}^{k} S_{i}-z\right) \cap X\right)=\bigoplus_{z \in Z} \bigoplus_{i=1}^{k}\left(S_{i}-z\right) \cap X=\bigoplus_{i=1}^{k} \bigoplus_{z \in Z}\left(S_{i}-z\right) \cap X=\bigoplus_{i=1}^{k} \phi\left(S_{i}\right)$.

For (3), observing that $(S-z) \cap X=S \cap(X+z)-z$, one concludes that

$A(\phi(S))=A\left(\bigoplus_{z \in Z}[S \cap(X+z)-z]\right) \leq \sum_{z \in Z} A(S \cap(X+z))=A(S) \cdot \tilde{d}_{\mathcal{F}}(S)$.

Instead of proving a lower bound on $\alpha^{\circ}(X)$, we shall prove one for $\alpha^{\circ}(-X+a)$, with a suitably chosen $a$. Since $\alpha^{\circ}(X)$ is invariant under affine transformations of $\mathbb{R}^{2}, \alpha^{\circ}(-X+a)=\alpha^{\circ}(X)$. For typographical reasons, set $X_{a}=-X+a$.

Consider, as before, any finite set $K \subset \mathbb{R}^{2}$ of odd cardinality, and let $Y_{a}=X_{a}+K$. On the one hand, by Claim 3.1 $(3), \quad A\left(\phi\left(Y_{a}\right)\right) \leq \tilde{d}_{\mathcal{F}}\left(Y_{a}\right) \cdot A\left(Y_{a}\right)$. On the other hand, by Claim 3.1 $(2) \&(1)$, $\phi\left(Y_{a}\right)=\phi\left(\bigoplus_{k \in K}\left(X_{a}+k\right)\right)=\bigoplus_{k \in K} \phi\left(X_{a}+k\right)=\bigoplus_{k \in K} X=X$. Thus,

$$
\tilde{d}_{\mathcal{F}}\left(Y_{a}\right) \cdot A\left(Y_{a}\right) \geq A\left(\phi\left(Y_{a}\right)\right)=A(X) \quad \Longrightarrow \quad \frac{A\left(Y_{a}\right)}{A\left(X_{a}\right)} \geq \tilde{d}_{\mathcal{F}}\left(Y_{a}\right)^{-1} .
$$

It remains to choose the translation vector $a$ as to minimize $\tilde{d}_{\mathcal{F}}\left(Y_{a}\right)$. Getting back to the discussion of Section 2.2, a simple averaging argument shows that for a random uniform $a \in Q_{n}$, the expected value of $\tilde{d}_{\mathcal{F}}\left(Y_{a}\right)$ gets arbitrarily close to $\tilde{d}_{\mathcal{F}}\left(Q_{n}\right)$ as $n$ tends to infinity. Keeping in mind the definition of $\rho_{\mathcal{F}}$, this implies in turn that there is a sequence of $a$ 's such that $\tilde{d}_{\mathcal{F}}\left(Y_{a}\right)$ approaches $\rho_{\mathcal{F}}$. Minimizing over all legal odd covers $\mathcal{F}$, one concludes that the infimum of $\tilde{d}_{\mathcal{F}}\left(Y_{a}\right)$ over $a \in \mathbb{R}^{2}$ is at most $\theta^{\circ}(X)$.

To demonstrate the usefulness of Lemma 3.1, assume that there is a tiling of $\mathbb{R}^{2}$ by translates of $X$. Then, $\theta^{\circ}(X)=1$, implying $\alpha^{\circ}(X)=1$. This yields the aforementioned result about the non-compressibility of the square and the centrally symmetric hexagon.

Further, assume that $X$ is a triangle $(a, b, c)$. Let $\Lambda$ be the lattice spanned by $\left\{\frac{1}{2}(b-a), \frac{1}{2}(c-a)\right\}$. Then, $\mathcal{F}=\{X+z\}_{z \in \Lambda}$ is an odd cover of $\mathbb{R}^{2}$ covering each point in the plane either 1 or 3 times, with $\rho(\mathcal{F})=2$. This implies $\alpha^{\circ}(X) \geq \frac{1}{2}$, matching the optimal bound of [1].

\section{Odd Covers by Stripe Patterns}

A stripe pattern is a (non-singular) affine image of the set $\left\{(x, y) \in \mathbb{R}^{2} \mid\lfloor y\rfloor\right.$ is even $\}$. I.e., it is an infinite set of parallel stripes of equal width $w$, such that the distance between any two adjacent stripes is $w$ as well (see Figure 1). The direction of a stripe pattern is, expectedly, the direction of a boundary line of any stripe in it. The width of the stripe pattern is the $w$ as above.

We start with the following simple but useful observation about stripe patterns. The easy verification is left to the reader. 


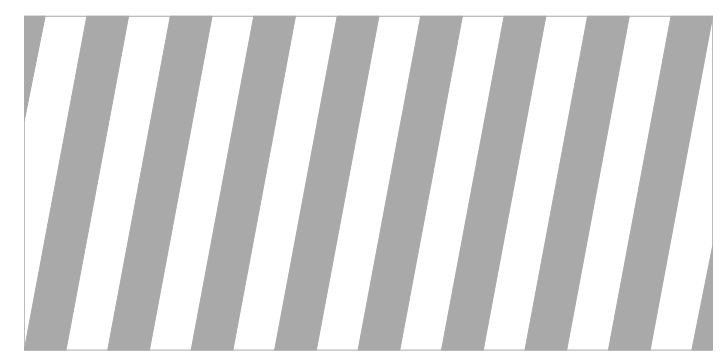

Figure 1: A stripes pattern

Observation 4.1. Let $S$ be a stripe pattern, and let $\ell$ and $r$ be the two lines delimiting one of the stripes in $S$. Then, for every $a \in \ell, b \in r$, and $v=b-a$, it holds that:

1. $S \stackrel{\circ}{+}\{0, v\}=S \oplus(S+v)=\mathbb{R}^{2}$.

2. $S+\left\{0, \frac{1}{2} v\right\}=S \oplus\left(S+\frac{1}{2} v\right)$ is a stripe pattern with the same direction as $S$, whose width is equal to half of the width of $S$.

The main result of this section is:

Lemma 4.1. Let $S_{1}, \ldots, S_{k}$ be stripe patterns with pairwise distinct directions, and let $T=S_{1} \oplus$ $\cdots \oplus S_{k}$. Then, there exists a finite (and efficiently computable) set of vectors $U \subset \mathbb{R}^{2},|U| \leq 2^{k-1}$, such that $T \stackrel{+}{+} U=\bigoplus_{u_{i} \in U}\left(T+u_{i}\right)=\mathbb{R}^{2}$, up to a set of measure 0 .

It will be technically more convenient to prove the following more general statement:

Lemma 4.2. Let $S_{1}, \ldots, S_{k}$ be stripe patterns with pairwise distinct directions, and let $\left\{Z_{i}\right\}_{i=1}^{k}$ be a family of finite nonempty subsets of $\mathbb{R}^{2}$, with $Z_{1}=\{0\}$. Let $T=\bigoplus_{i=1}^{k}\left(S_{i}+Z_{i}\right)$. Then, as before, there exists a finite (and efficiently computable) set of vectors $U \subset \mathbb{R}^{2},|U| \leq 2^{k-1}$, such that $T \stackrel{+}{+} U=\bigoplus_{u_{i} \in U}\left(T+u_{i}\right)=\mathbb{R}^{2}$, up to a set of measure 0 .

Lemma 4.1 follows from Lemma 4.2 by setting $Z_{i}=\{0\}$ for all $i \geq 2$.

Notice the special role of $S_{1}$ in the statement of Lemma 4.2 . In fact, the condition $Z_{1}=\{0\}$ is essential even for $k=1$. It is easy to verify that, using the notation of Observation 4.1, no finite set of translates of the set $T=S_{1}+\left\{0, \frac{2}{3} v\right\}$ can oddly cover the plans ${ }^{3}$.

Proof. (of Lemma 4.2) For every $i=1,2, \ldots, k$, let $\ell_{i}$ and $r_{i}$ denote the two parallel lines delimiting some stripe in $S_{i}$. By the assumptions of the Lemma, for different $i$ 's these have different directions, and therefore intersect.

The proof proceeds by induction on $k$.

For $k=1$, the statement follows from Observation 4.1(1).

For $k=2$, let $a$ and $b$ be the intersection points of $\ell_{1}$ and $r_{1}$ with $\ell_{2}$, respectively. Setting $v_{2}=b-a$, we have $S_{1}+\left\{0, v_{2}\right\}=\mathbb{R}^{2}$, by Observation 4.1.(1). Moreover, since $v_{2}$ has the same direction as of $S_{2}$, we have $S_{2}+\left\{0, v_{2}\right\}=\emptyset$. Keeping this in mind we have:

$$
\begin{gathered}
T \stackrel{\circ}{+}\left\{0, v_{2}\right\}=\left(S_{1} \oplus\left(S_{2}+Z_{2}\right)\right) \stackrel{\circ}{+}\left\{0, v_{2}\right\}=\left(S_{1} \stackrel{\circ}{+}\left\{0, v_{2}\right\}\right) \oplus\left(S_{2}+Z_{2}+\left\{0, v_{2}\right\}\right)= \\
=\mathbb{R}^{2} \oplus\left(S_{2} \stackrel{\circ}{+}\left\{0, v_{2}\right\} \stackrel{+}{+} Z_{2}\right)=\mathbb{R}^{2} \oplus\left(\emptyset \stackrel{\circ}{+} Z_{2}\right)=\mathbb{R}^{2} \oplus \emptyset=\mathbb{R}^{2} .
\end{gathered}
$$

\footnotetext{
${ }^{3}$ Perhaps expectedly, the same $T$ has also the complementary extremal property: $T+\left\{0, \frac{1}{3} v, \frac{2}{3} v\right\}=\emptyset$.
} 
For $k>2$, we proceed as follows. Let $v_{k}$ be the (well-defined) vector such that, on the one hand, $\ell_{k}+v_{k}=r_{k}$, and on the other hand, $\ell_{1}+2 v_{k}=r_{1}$. Observation 4.1 (1) implies that $S_{k}+\left\{0, v_{k}\right\}=\mathbb{R}^{2}$, and hence $\left(S_{k} \stackrel{\circ}{+} Z_{k}\right) \stackrel{\circ}{+}\left\{0, v_{k}\right\}$ equals $\mathbb{R}^{2} \stackrel{\circ}{+} Z_{k}$, which is either $\emptyset$ or $\mathbb{R}^{2}$, depending on the parity of $Z_{k}$. Observation 4.1 (2) implies that $S_{1}+\left\{0, v_{k}\right\}$ is a stripe pattern with the same direction as $S_{1}$, and half its width. Consequently, $\left(S_{1} \stackrel{\circ}{+}\left\{0, v_{k}\right\}\right) \oplus\left(\left(S_{k} \stackrel{\circ}{+} Z_{k}\right) \stackrel{\circ}{+}\left\{0, v_{k}\right\}\right)$ is a stripe pattern with the same direction as $S_{1}$ and half its width as well.

Consider now the set $T^{\prime}=T \stackrel{\circ}{+}\left\{0, v_{k}\right\}=T \oplus\left(T+v_{k}\right)$. Using the properties of the operators $\oplus$

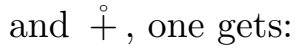

$$
T^{\prime}=T \stackrel{\circ}{+}\left\{0, v_{k}\right\}=\left(\bigoplus_{i=1}^{k}\left(S_{i} \stackrel{\circ}{+} Z_{i}\right)\right) \stackrel{\circ}{+}\left\{0, v_{k}\right\}=\bigoplus_{i=1}^{k}\left(S_{i} \stackrel{\circ}{+} Z_{i} \stackrel{\circ}{+}\left\{0, v_{k}\right\}\right)
$$

As we have just seen, the $\oplus$ of the first and the $k^{\prime}$ th terms of the latter sum is a stripe pattern $S_{1}^{\prime}$ with the same direction as $S_{1}$. Thus, setting $Z_{i}^{\prime}=Z_{i} \stackrel{\circ}{+}\left\{0, v_{k}\right\}$, one arrives at

$$
T^{\prime}=S_{1}^{\prime} \oplus \bigoplus_{i=2}^{k-1}\left(S_{i}+Z_{i}^{\prime}\right)
$$

By the induction hypothesis applied to $T^{\prime}$, there exists a finite set $U^{\prime} \subset \mathbb{R}^{2}$ such that $T^{\prime}+U^{\prime}=$ $\mathbb{R}^{2}$ up to a set of measure 0 . However,

$$
T^{\prime} \stackrel{\circ}{+} U^{\prime}=T \stackrel{\circ}{+}\left\{0, v_{k}\right\} \stackrel{\circ}{+} U^{\prime}=T \stackrel{\circ}{+}\left(U^{\prime} \stackrel{\circ}{+}\left\{0, v_{k}\right\}\right)
$$

Therefore, setting $U=U^{\prime} \stackrel{\circ}{+}\left\{0, v_{k}\right\}$, one concludes that $T \stackrel{+}{+}=T^{\prime} \stackrel{\circ}{+} U^{\prime}=\mathbb{R}^{2}$. This completes the construction of the desired set $U$.

It remains to estimate the size of $U$. The recursive definition $U=U^{\prime}+\left\{0, v_{k}\right\}$ for $k>2$, combined with the base cases $|U|=2^{k-1}$ for $k=1,2$, implies the desired bound: $|U| \leq 2^{k-1}$.

\section{Odd Covers by Rational Polygons: A Special Case}

In this section we prove our main theorem for the special case of rational polygons with no two parallel edges.

Given a rational polygon $P$, let $P_{\mathrm{INT}}$ be the integer polygon with minimal area affinely equivalent to $P$, and let $A_{\mathrm{INT}}(P)=A\left(P_{\mathrm{INT}}\right)$ be its area.

Theorem 5.1. Let $P$ be a rational polygon with $k$ vertices, and no parallel edges. Then, there exists a bounded degree odd cover $\mathcal{F}$ of $\mathbb{R}^{2}$ by translates of $P$ with density $\rho(\mathcal{F}) \leq A_{\mathrm{INT}}(P) \cdot 2^{k-1}$. Consequently, $\alpha^{\circ}(P) \geq A_{\mathrm{INT}}(P)^{-1} \cdot 2^{-(k-1)}$.

Before starting with the proof, we need one more observation about the structure of $\oplus$-sums of stripe patterns. For $i=1, \ldots r$, let $L_{i}$ be an affine image of the family of parallel lines $\{(x, y) \in$ $\left.\mathbb{R}^{2} \mid y \in \mathbb{Z}\right\}$. Respectively, let $S_{i}$ be a stripe pattern whose boundary is $L_{i}$. (Notice that there are exactly two such stripe patterns: $S_{i}$ and its complement $\bar{S}_{i}=\mathbb{R}^{2} \backslash S_{i}$.) Assume that $S_{1}, \ldots, S_{r}$ have pairwise distinct directions. The union of all these lines $\bigcup_{i=1}^{r} L_{i}$ partitions $\mathbb{R}^{2}$ into pairwise disjoint open cells, each cell being a convex polygon. Call two cells adjacent if they share a 1-dimensional edge.

It is a folklore to show that the cells of $\mathbb{R}^{2} \backslash \bigcup_{i=1}^{r} L_{i}$ can be 2-colored in such a way that any two adjacent cells have different colors. 
Claim 5.1. Let $T$ be the union of all cells of $\mathbb{R}^{2} \backslash \bigcup_{i=1}^{r} L_{i}$ in one color class. Then, (up to the 0-measure boundary of $T$, i.e., $\left.\bigcup_{i=1}^{r} L_{i}\right)$ either $T=S_{1} \oplus \cdots \oplus S_{r}$, or $T=\mathbb{R}^{2} \backslash\left(S_{1} \oplus \cdots \oplus S_{r}\right)=$ $\bar{S}_{1} \oplus S_{2} \oplus \cdots \oplus S_{r}$.

The claim is rather obvious, and can be formally verified, e.g., by induction on $r$. The full details are left to the reader (see Figure 2 for an illustration).
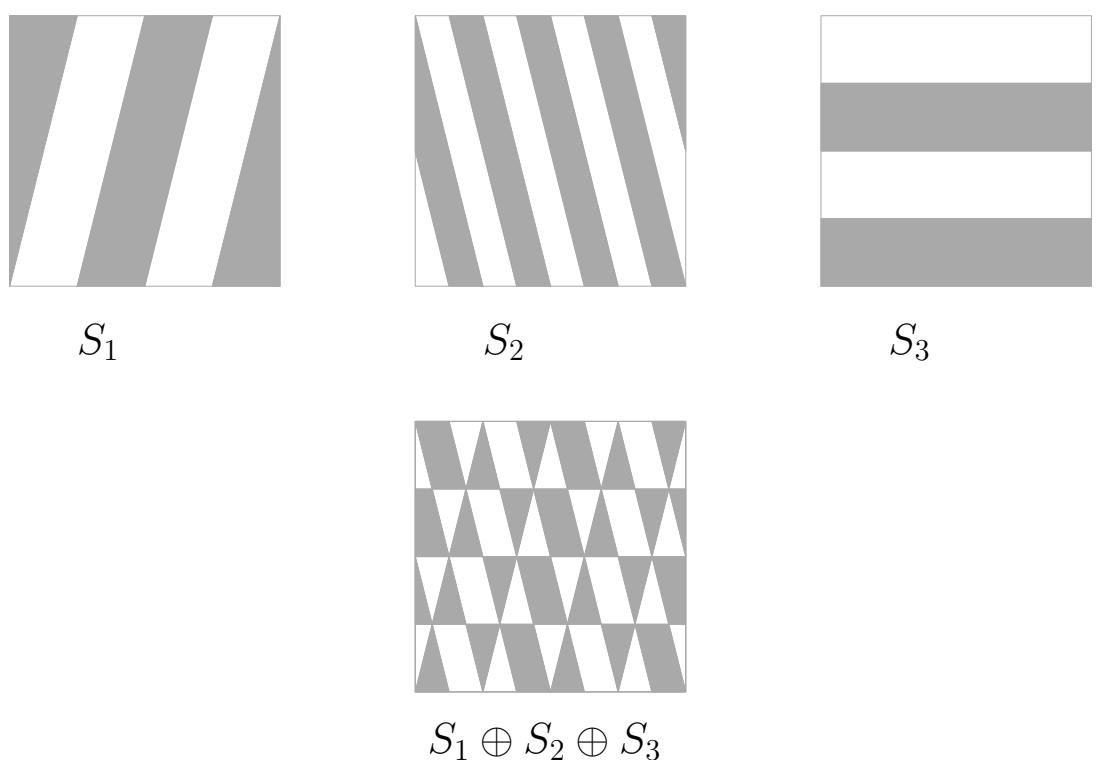

Figure 2: $\oplus$-sum of three stripes patterns

Proof. (of Theorem 5.1) Keeping in mind that both $\theta^{\circ}(P)$ and $\alpha^{\circ}(P)$ are affine invariants, one may assume without loss of generality that $P=P_{\mathrm{INT}}$, and that the origin $O=(0,0)$ is a vertex of $P$. Then, all the vertices of $P$ belong to $\mathbb{Z}^{2}$. Observe also that some of the edges of $P$ must contain an even number of integer lattice points. Otherwise, the coordinates of the vertices of $P$ would all have the same parity, i.e., they would all be even. Scaling such an all-even $P$ by a factor of $\frac{1}{2}$ would have yielded a smaller integer polygon affinely equivalent to $P$, contrary to the definition of $P_{\text {INT }}$.

We claim that $P+\mathbb{Z}^{2}$ is equal to $S_{1} \oplus \ldots \oplus S_{r}$, where $S_{1}, \ldots, S_{1}$ are stripe patterns with pairwise distinct directions, and $r$ is at most the number of vertices (=edges) of $P$. Once this claim is established, the rest easily follows.

Indeed, assuming that the claim holds, by Lemma 4.1 there exists $U \subset \mathbb{R}^{2}$ with $|U| \leq 2^{r-1}$ such that $\left(P \stackrel{\circ}{+} \mathbb{Z}^{2}\right) \stackrel{+}{+}=\mathbb{R}^{2}$. Equivalently, the (multi-) family of sets $\mathcal{F}=\{P+z+u\}_{z \in \mathbb{Z}^{2}, u \in U}$ is an odd cover of the plane. To employ the Odd Cover Lemma 3.1, one needs to estimate the density of this cover. Observe that $\{P+z\}_{z \in \mathbb{Z}^{2}}$ has a bounded maximal degree (being the maximal number of integer lattice points in any translate of $P$ ), while its average density is $A(P)$, as mentioned in Claim 2.1. Therefore, the maximal degree of $\mathcal{F}$ is at most $|U|$ times the maximal degree of the cover $\{P+z\}_{z \in \mathbb{Z}^{2}}$, while $\rho(\mathcal{F})$, the average degree of $\mathcal{F}$, is precisely $A(P) \cdot|U| \leq$ $A(P) \cdot 2^{k-1}$. Hence, $\theta^{\circ}(P) \leq \rho(\mathcal{F}) \leq A(P) \cdot 2^{k-1}$. Applying the Odd Cover Lemma 3.1 one gets $\alpha^{\circ}(P) \geq \theta^{\circ}(P)^{-1} \geq A(P)^{-1} \cdot 2^{-(k-1)}$, as needed.

Thus, it is sufficient to show that $P \stackrel{\circ}{+} \mathbb{Z}^{2}$ is equal to $S_{1} \oplus \ldots \oplus S_{r}$ as above. In the remainder of this section, we shall focus on proving this claim. The argument goes as follows. 
Let $E(P)$ denote the set of all edges of $P$. For $e \in E(P)$, let $L_{e}$ be the set of all lines parallel to $e$ that contain points of $\mathbb{Z}^{2}$. Clearly, $L_{e}$ is a discrete set of lines as in Claim 5.1 . Consider a point $x \in \mathbb{R}^{2}$. It belongs to $P \stackrel{\circ}{+} \mathbb{Z}^{2}$ exactly when $\left|P \cap\left(x-\mathbb{Z}^{2}\right)\right|$ is odd. Unless $x \in \bigcup_{e \in E(P)} L_{e}$, every point $x^{\prime}$ in a sufficiently small neighborhood of $x$ will satisfy $\left|P \cap\left(x-\mathbb{Z}^{2}\right)\right|=\left|P \cap\left(x^{\prime}-\mathbb{Z}^{2}\right)\right|$. Therefore, $P \stackrel{\circ}{+} \mathbb{Z}^{2}$ is a union of cells of $\mathbb{R}^{2} \backslash \bigcup_{e \in E(P)} L_{e}$.

Call an edge $e$ of $P$ active if it contains an even number of integer lattice points, and passive otherwise. Respectively, if $e$ is active, all the lines in $L_{e}$ are called active, and if it is passive, the lines in $L_{e}$ are called passive.

Let $C_{1}$ and $C_{2}$ be two adjacent cells in $\mathbb{R}^{2} \backslash \bigcup_{e \in E(P)} L_{e}$ separated by a line $\ell \in L_{e}$ for some edge $e$ of $P$. We claim that if $e$ is active, then exactly one of $C_{1}$ and $C_{2}$ is contained in $P \stackrel{\circ}{+}$, and if $e$ is passive, then either both are contained in $P \stackrel{+}{+} \mathbb{Z}^{2}$, or none of them is.

Indeed, let $I \subset \ell$ denote the common 1-dimensional edge of $C_{1}$ and $C_{2}$. Observe that the only members in the family $\mathcal{F}=\{P+z\}_{z \in \mathbb{Z}}$ that distinguish between $C_{1}$ and $C_{2}$, that is, contain exactly one of the two, are those that contain $I$ in their boundary. To get a clearer picture of this subfamily, let $J=[p, q] \subset \ell$ be the smallest interval with integer endpoints containing $I$. Notice that $I$ has no integer points in its interior. Let us view $e$ as a 1-dimensional interval $\left[s_{e}, t_{e}\right) \subset \mathbb{R}^{2}$, parallel to, and having the same orientation as, $J$. Then, $P+z$ contains $I$ if and only if $p \in e+z$. Or, equivalently, $p-z \in e$.

This means that when $e$ is active (i.e., it contains an even number of points in $\mathbb{Z}^{2}$ ), $I$ is covered by an odd number of $(P+z)$ 's, and when $e$ is passive, it is covered by an even number of $(P+z)$ 's. Consequently, in the former case the degrees of cover of the cells $C_{1}$ and $C_{2}$ by $\mathcal{F}$ have a different parity, whereas in the latter case the parities are equal. Thus, when $e$ is active, $P+\mathbb{Z}^{2}$ distinguishes between $C_{1}$ and $C_{2}$, and when it is passive, it does not. As claimed.

Let $A E(P)$ be the (nonempty!) set of active edges of $P$. The conclusion is that $P+\mathbb{Z}^{2}$ is a union of cells of $\mathbb{R}^{2} \backslash \bigcup_{e \in A E(P)} L_{e}$, satisfying the assumptions of Claim 5.1. Hence, $P \stackrel{+}{+} \mathbb{Z}^{2}$ is a $\oplus$-sum of stripe patterns, as desired. This completes the proof of Theorem 5.1 .

The assumption that $P$ has no parallel edges was needed to justify the (tacit) assumption that for every line $\ell \in \bigcup_{e \in E(P)} L_{e}$, there is a unique edge $e$ such that any translate of $P$ may have contained in $\ell$. When there are parallel edges, most of the argument still applies, however, it may fail at one fine point. The contributions of parallel edges may cancel out, leaving no active lines, and resulting in $P \stackrel{\circ}{+} \mathbb{Z}^{2}=\emptyset$. Unfortunately, this situation indeed does occur for some rational polygons $P$, for example, for the centrally symmetric ones. To overcome this problem, a more refined family of translates will be constructed.

\section{A Theorem About $\mathbb{Z}_{2}$-valued Functions on Integer Lattices}

We shall need the following result of an independent interest. It will be proven here for any dimension $d$, but used in Section 7 only with $d=2$.

Let $\mathcal{A}$ be a family of finite subsets of $\mathbb{Z}^{d}$. A function, or, rather, a weighting, $\mathfrak{F}: \mathbb{Z}^{d} \rightarrow \mathbb{Z}_{2}$, will be called stable with respect to $\mathcal{A}$, if for any $A \in \mathcal{A}$, all integer translates of $A$ have the same $\mathfrak{F}$-weight. That is, the value of $\mathfrak{F}(A+p)=\bigoplus_{x \in A+p} \mathfrak{F}(x)$, does not depend on the choice of $p \in \mathbb{Z}^{d}$, but solely on $\left.A\right|^{4}$ Further, call $\mathfrak{F}$ 0-stable with respect to $\mathcal{A}$, if it is stable, and moreover, for every $A \in \mathcal{A}, \mathfrak{F}(A)=0$. For example, if the function $\mathfrak{F}$ is everywhere 0 , then it is 0 -stable with respect

\footnotetext{
${ }^{4}$ In this section, the operator $\oplus$ that was originally defined on sets, will be sometimes applied to points. For consistency, regard points as single-element sets.
} 
to any family $\mathcal{A}$. If it is everywhere 1 , it is stable with respect to any family $\mathcal{A}$, and 0 -stable if $\mathcal{A}$ consists only of sets of even cardinality.

Theorem 6.1. Let $\mathcal{A}$ be a (possibly infinite) family of non-empty finite subsets of $\mathbb{Z}^{d}$, and $\mathcal{A} \neq \emptyset$. There exists a function $\mathfrak{F}: \mathbb{Z}^{d} \rightarrow \mathbb{Z}_{2}$ that is stable, but not 0 -stable, with respect to $\mathcal{A}$.

Proof. We start with the 1-dimensional case, introducing the key construction to be used in all dimensions.

Case $d=1$

We define a family $\left\{\mathfrak{f}_{k}\right\}_{k=0}^{\infty}$ of functions from $\mathbb{Z}$ to $\mathbb{Z}_{2}$ in the following recursive manner. We will show that one of this functions is the desired function $\mathfrak{F}$ :

$\mathfrak{f}_{0}$ is identically 1 ;

For $k>0, \quad \mathfrak{f}_{k}(0)=1$, and $\mathfrak{f}_{k}(t)=\mathfrak{f}_{k}(t-1) \oplus \mathfrak{f}_{k-1}(t-1) \cdot 5$

For example, $\mathfrak{f}_{1}(t)$ is 1 if $t$ is even, and 0 otherwise. The next one, $\mathfrak{f}_{2}(t)$, is 1 if $t \equiv 0,3(\bmod 4)$, and 0 otherwise. Observe that the repeated application of the recursive formula yields for any $c \in \mathbb{N}$,

$$
\mathfrak{f}_{k}(t+c)=\mathfrak{f}_{k}(t) \oplus \bigoplus_{i=0}^{c-1} \mathfrak{f}_{k-1}(t+i) .
$$

Claim 6.1. If $\mathfrak{f}_{k-1}$ is 0 -stable with respect to a finite $A \subseteq \mathbb{Z}$, then $\mathfrak{f}_{k}$ is stable with respect to $A$.

Indeed, it suffices to show that for any $p \in \mathbb{Z}, \mathfrak{f}_{k}(A+p+1)=\mathfrak{f}_{k}(A+p)$. By definition of $\mathfrak{f}_{k}$,

$\mathfrak{f}_{k}(A+p+1)=\bigoplus_{t \in A+p+1} \mathfrak{f}_{k}(t)=\bigoplus_{t \in A+p+1} \mathfrak{f}_{k}(t-1) \oplus \bigoplus_{t \in A+p+1} \mathfrak{f}_{k-1}(t-1)=\mathfrak{f}_{k}(A+p) \oplus \mathfrak{f}_{k-1}(A+p)$

Since $\mathfrak{f}_{k-1}(A+p)=0$ by assumptions of the claim, one concludes that $\mathfrak{f}_{k}(A+p+1)=\mathfrak{f}_{k}(A+p)$.

Claim 6.2. For any $k \geq 1, \mathfrak{f}_{k}(t)=0$ for $1 \leq t \leq k$.

Indeed, apply induction on $k$. For $k=1, \mathfrak{f}_{1}(1)=\mathfrak{f}_{1}(0) \oplus \mathfrak{f}_{0}(0)=1 \oplus 1=0$. For $k>1$, using (5), one concludes that for any $t$ in the range,

$$
\mathfrak{f}_{k}(t)=\mathfrak{f}_{k}(0) \oplus \mathfrak{f}_{k-1}(0) \oplus \mathfrak{f}_{k-1}(1) \oplus \ldots \oplus \mathfrak{f}_{k-1}(t-1)=1 \oplus 1 \oplus 0 \oplus \ldots \oplus 0=0 .
$$

We proceed to show that one of $\mathfrak{f}_{k}$ 's satisfies the requirements of the theorem. Observe that $\mathfrak{f}_{0}$ is stable with respect to $\mathcal{A}$. By Claim 6.1, either there exists $k \geq 0$ such that $\mathfrak{f}_{k}$ is stable, but not 0 -stable (precisely as desired), or all $\mathfrak{f}_{k}$ 's are 0 -stable. However, the latter situation does not occur. Consider any nonempty $A \in \mathcal{A}$, and let $a=\min (A), b=\max (A), r=b-a$. Then, by Claim 6.2 . $\mathfrak{f}_{r}(A-a)=1$, and thus $\mathfrak{f}_{r}$ is not 0 -stable. This completes the case $d=1$.

\section{General Case}

Let $L$ be a linear function (without a constant term) from $\mathbb{Z}^{d}$ to $\mathbb{Z}$ that satisfies two requirements. The first requirement is that the coefficient of $x_{1}$ in $L$ is 1 . The second requirement is that for some $A \in \mathcal{A}, L$ attains a minimum on $A$ at a unique point. Such $L$ 's exist. E.g., assuming that $A$ can

\footnotetext{
${ }^{5}$ Observe that this recursive formula defines $\mathfrak{f}_{k}(t)$ for both positive and negative values of $t$. More explicitly, for $t<0$ it becomes $\mathfrak{f}_{k}(t)=\mathfrak{f}_{k}(t+1) \oplus \mathfrak{f}_{k-1}(t)$, reducing either $k$ or $|t|$ just as for $t>0$.
} 
be translated to a subset of a of the cube $[0, r-1]^{d}$, the function $\sum_{i=1}^{d} r^{i-1} x_{i}$ is one-to-one on $A$ by the uniqueness of the base- $r$ representation, and so its minimum on $A$ is attained exactly once.

For $k \geq 0$ and $a \in \mathbb{Z}^{d}$, define $\mathfrak{F}_{k}(a)=\mathfrak{f}_{k}(L(a))$. Respectively, for a finite subset $A \subset \mathbb{Z}^{d}$, define $\mathfrak{F}_{k}(A)=\bigoplus_{a \in A} \mathfrak{F}_{k}(a)$.

The proof proceeds along the same lines as in the 1-dimensional case.

Claim 6.3. If $\mathfrak{F}_{k-1}$ is 0-stable with respect to a finite $A \subseteq \mathbb{Z}^{d}$, then $\mathfrak{F}_{k}$ is stable with respect to it.

It suffices to show that for any $p \in \mathbb{Z}^{d}$, and any unit vector $e \in \mathbb{Z}^{d}, \mathfrak{F}_{k}(A+p+e)=\mathfrak{F}_{k}(A+p)$. Let $L(e)=c$. If $c=0$, the statement is trivial. If $c<0$ the statement reduces to the case $c>0$ by considering $-e$ instead of $e$. Thus, without loss of generality, $c>0$. By (5), the linearity of $L$, and the first requirement on it,

$$
\begin{gathered}
\mathfrak{F}_{k}(A+p+e)=\bigoplus_{t \in A+p+e} \mathfrak{f}_{k}(L(t))=\bigoplus_{t \in A+p} \mathfrak{f}_{k}(L(t)+c)=\bigoplus_{t \in A+p} \mathfrak{f}_{k}(L(t)) \oplus \bigoplus_{i=0}^{c-1} \bigoplus_{t \in A+p} \mathfrak{f}_{k-1}(L(t)+i)= \\
=\mathfrak{F}_{k}(A+p) \oplus \bigoplus_{i=0}^{c-1} \bigoplus_{t \in A+p+i \cdot e_{1}} \mathfrak{f}_{k-1}(L(t))=\mathfrak{F}_{k}(A+p) \oplus \bigoplus_{i=0}^{c-1} \mathfrak{F}_{k-1}\left(A+p+i \cdot e_{1}\right) .
\end{gathered}
$$

Since $\mathfrak{F}_{k-1}$ is 0 -stable with respect to $A$, the second summand is 0 , and thus $\mathfrak{F}_{k}(A+p+e)=\mathfrak{F}_{k}(A+p)$. This concludes the proof of Claim 6.3 .

To conclude the proof of the theorem, observe that $\mathfrak{F}_{0}$ is stable with respect to $\mathcal{A}$, and thus, by Claim 6.3, either there exists $k \geq 0$ such that $\mathfrak{F}_{k}$ is stable, but not 0 -stable, precisely as desired, or all $\mathfrak{F}_{k}$ 's are 0-stable.

As before, the second possibility does not occur. Indeed, by the second requirement on $L$, there exists $A \in \mathcal{A}$ on which $L$ attains a unique minimum. Let $p \in A$ be the point on which the minimum is attained, and let $a \in \mathbb{Z}$ denote its value. Then, $L\left(A-a \cdot e_{1}\right)=L(A)-a$ is a subset of $[0, k]$ for some $k \in \mathbb{N}$, and 0 has a unique pre-image $p^{\prime}=p-a \cdot e_{1}$. By Claim 6.2 , $\mathfrak{F}_{k}\left(A-a \cdot e_{1}\right)=\mathfrak{f}_{k}(0) \oplus \bigoplus_{t \in A \backslash p^{\prime}} \mathfrak{f}_{k}(L(t))=1 \oplus 0=1$.

\section{The Main Theorem}

We can now prove the main theorem in full generality, making no assumption about parallel edges.

Theorem 7.1. Let $P$ be a rational polygon with $k$ distinct classes of parallel edges. Then, there exists a bounded degree odd cover $\mathcal{F}$ of $\mathbb{R}^{2}$ by translates of $P$ with density $\rho(\mathcal{F}) \leq A_{\mathrm{INT}}(P) \cdot 2^{k-1}$. Consequently, $\alpha^{\circ}(P) \geq A_{\mathrm{INT}}(P)^{-1} \cdot 2^{-(k-1)}$.

Proof. While the family of translates will in general be different from the one used in the proof of Theorem 5.1, the logical structure of the proof will be essentially identical. Let us re-examine this structure.

Assuming that $P=P_{I N T}$, the first and main goal is to construct a family $\mathcal{F}=\{P+z\}_{z \in Z}$, $Z \subseteq \mathbb{Z}^{2}$, such that $P \stackrel{+}{+}$ is a $\oplus$-sum of at most $k$ stripe patterns. (In the former proof, $Z$ was just $\mathbb{Z}^{2}$.) A close reading of the proof of Theorem 5.1 reveals that in order to prove this fact about $\mathcal{F}$, it is sufficient to show that $\mathcal{F}$ has a certain property. To formulate it we need some definitions.

Let $D(P)$ be the set of all the classes of parallel edges of $P$, or simply the directions of $P$. For every $d \in D(P)$, let $L_{d}$ be the set of all lines in $\mathbb{R}^{2}$ in the direction of $d$ that contain integer lattice points. As before, each $L_{d}$ is a discrete set of parallel lines with equal distances between any two consecutive ones. 
Consider the arrangement of lines $\bigcup_{d} L_{d}$. Observe that since $Z \subseteq \mathbb{Z}^{2}$, for any $(P+z) \in \mathcal{F}$, and any open cell $C$ of $\mathbb{R}^{2} \backslash \bigcup_{d} L_{d}$, either $C \subseteq(P+z)$, or $C \cap(P+z)=\emptyset$.

Property 7.1. Let $P, D(P), Z, \mathcal{F}, \bigcup_{d} L_{d}$ be as above. Further, let I denote an edge of the arrangement $\bigcup_{d} L_{d}$ (i.e., a common 1-dimensional boundary of two adjacent cells) that lies on a line $\ell \in L_{d}, d \in D(P)$. The family $\mathcal{F}$ has the desired property if:

1. For any edge $I$ of the arrangement $\bigcup_{d} L_{d}$ as above, the parity of the number of sets in $\mathcal{F}$ whose boundary contains I depends solely on the corresponding direction $d$.

2. Moreover, there exists $d \in D(P)$ such that this parity is odd. We call such a direction active, and denote the set of all active directions by $A D(P)$.

Once Property 7.1 is established for $\mathcal{F}=\{P+z\}_{z \in Z}$, the argument from the proof of Theorem 5.1 implies that $P+Z$ is a (nonempty) union of cells of $\mathbb{R}^{2} \backslash \bigcup_{d \in A D(P)} L_{d}$ that satisfy the assumptions of Claim 5.1. Applying Claim 5.1, one concludes that $P \stackrel{+}{+}$ is equal to $S_{1} \oplus \ldots \oplus S_{r}$ for some stripe patterns $S_{1}, \ldots, S_{r}$, and $r=|A D(P)|$, the number of active directions, is at most $|D(P)|=k$.

Once the main goal is achieved, the rest is easy. Lemma 4.1 is used to conclude that there exists a finite set $U \subset \mathbb{R}^{2}$ with $|U| \leq 2^{r-1}$, such that $(P+Z)+U=\mathbb{R}^{2}$. Equivalently, the (multi-) family of sets $\mathcal{F}=\{P+z+u\}_{z \in Z, u \in U}$ is an odd cover of the plane. Since $Z$ is a subset of $\mathbb{Z}^{2}$, the density of this odd cover is at most $A_{\mathrm{INT}}(P) \cdot|U| \leq A_{\mathrm{INT}}(P) \cdot 2^{k-1}$. (For the appearance of $A_{\mathrm{INT}}$, consult Claim 2.1.) Finally, by the Odd Cover Lemma 3.1, one concludes that $\alpha^{\circ}(P) \geq A_{\mathrm{INT}}(P)^{-1} \cdot 2^{-(k-1)}$, establishing the theorem.

In view of the above, in order to prove Theorem 7.1, it suffices to construct $Z \subseteq \mathbb{Z}^{2}$ such that $\mathcal{F}=\{P+z\}_{z \in Z}$ has Property 7.1. The remaining part of this section is dedicated to constructing such $Z$, and proving that $\mathcal{F}$ has the required property.

The set of translates $Z$ is constructed as follows. Assume that $P=P_{I N T}$. In particular, the vertices of $P$ are in $\mathbb{Z}^{2}$. For every direction $d \in D(P)$, define the vector $v_{d} \in \mathbb{Z}^{2}$ as the difference between (any) pair of two consecutive integer lattice points on (any) line in $L_{d}$, the set of all lines in direction $d$ through an integer lattice point.

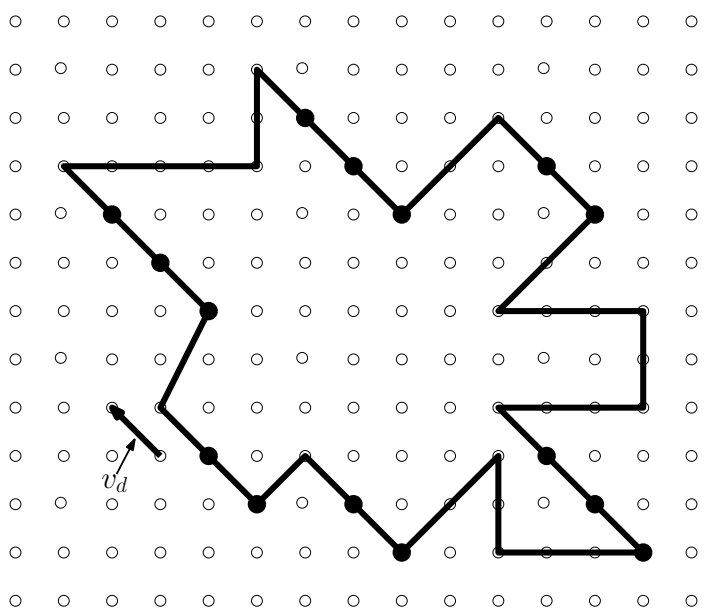

Figure 3: The points of $A_{d}$ are the filled discs in the picture

Let $A_{d}$ be the set of all integer lattice points $z$ on the boundary of $P$ such that both $z$ and $z+v_{d}$ lie on an edge of $P$ in the direction $d$ (see Figure 3). Let $\mathcal{A}=\left\{-A_{d}\right\}_{d \in D(P)}$. By Theorem 6.1, there 
exists a $\mathbb{Z}_{2}$-weighting $\mathfrak{F}$ of $\mathbb{Z}^{2}$ that is stable, but not 0 -stable, with respect to $\mathcal{A}$. Define $Z$ as the support of $\mathfrak{F}$, i.e., $Z=\{z \mid \mathfrak{F}(z)=1\}$. Finally, define $\mathcal{F}=\{P+z\}_{z \in Z}$. Our goal is to show that the family $\mathcal{F}=\{P+z\}_{z \in Z}$ has Property 7.1.

Call a direction $d$ of an edge of $P$ active if $\mathfrak{F}\left(-A_{d}\right)=1$, and passive if $\mathfrak{F}\left(-A_{i}\right)=0$.

We claim that a point $p \in \mathbb{Z}^{2}$ belongs to an odd number of sets in $\left\{A_{d}+z\right\}_{z \in Z}$ if $d$ is active, and to an even number of those sets if $d$ is passive. Indeed, the number of solutions of the equation $a+z=p$, where $a \in A_{d}, z \in Z$, is precisely the size of $\left(-A_{d}+p\right) \cap Z$, and hence its parity is $\mathfrak{F}\left(-A_{d}+p\right)=\mathfrak{F}\left(-A_{d}\right)$, as desired.

Let $I \subset \ell \in L_{d}$, for some $d$, be an edge in the arrangement of lines $\bigcup_{d \in D(P)}$. Notice that $I$ cannot contain integer lattice points in its (relative) interior. There exists two consecutive integer lattice points $p$ and $q$ on $\ell$ such that $I$ is contained in the line segment $J=[p, q] \subset \ell$. Observe that $q-p$ is either $v_{d}$ or $-v_{d}$; assume w.l.o.g., that $q-p=v_{d}$.

We claim that the parity of the number of sets from $\mathcal{F}=\{P+z\}_{z \in Z}$ whose boundary contains $J$ is odd if $d$ is active, and even if it is passive. Indeed, $J$ is contained in the boundary of $(P+z)$, $z \in Z$, if and only if $\left(A_{d}+z\right)$ contains $p$. As we have already seen, the parity of the number of such sets is odd if and only if $d$ is active. In particular, it depends only on $d$, and not on $I$, as desired. Moreover, by Theorem 6.1, there exists at least one active direction $d$.

This concludes the verification of Property 7.1 for the constructed family $\mathcal{F}=\{P+z\}_{z \in Z}$, which in turn concludes the proof of Theorem 7.1 .

Notice that the above proof makes no use of the connectivity of $P$ nor of the connectivity of its boundary. Thus, as it has been already mentioned in the Introduction, Theorem 7.1 applies equally well to any compact figure in $\mathbb{R}^{2}$ with non-empty interior, piecewise linear boundary, and finite number of vertices, all of which are rational.

\section{References}

[1] R. Pinchasi, Points covered an odd number of times by translates, Amer. Math. Monthly 121 (2014), no. 7, 632-636.

[2] A. Oren, I. Pak, R. Pinchasi, On the odd area of planar sets, Discrete Comput. Geom. 55 (2016), no. 3, 715-724.

[3] C.A. Rogers, Packings and coverings, Cambridge Tracts in Mathematics and Mathematical Physics, No. 54, Cambridge 1964.

[4] The International Mathematics Tournament of the Towns, Fall 2009, available at http://www.math.toronto.edu/oz/turgor/archives/TT2009F_JAproblems.pdf. 